\title{
EL PAPEL DEL MOVIMIENTO OLÍMPICO EN LA PROMOCIÓN DE LOS DERECHOS HUMANOS ${ }^{1}$
}

\author{
Alexandre MESTRE \\ Miembro Independiente del Comité de Gobierno y Cumplimiento de la UEFA \\ Portugal \\ alexandremestre@hotmail.com
}

Fecha de recepción: septiembre 2010

Fecha de aceptación: noviembre 2020

http://doi.org/10.15366/citius2020.13.2.001

\section{Resumen:}

La Organización de las Naciones Unidas (ONU) define los derechos humanos como «las garantías legales universales que protegen a las personas y los grupos contra acciones u omisiones de los gobiernos que interfieran con la dignidad humana». Sin embargo, el comportamiento de ciertas organizaciones deportivas, con cierta frecuencia vulneran estos derechos. El Movimiento Olímpico debe tener un papel en la promoción y la defensa de los derechos humanos. La Carta Olímpica es el único texto legal internacional del mundo que reconoce expresamente el deporte como derecho humano. La Agenda 2020 y las revisiones periódicas de la Carta Olímpica, muestran claramente el liderazgo del COI en la promoción y la defensa de los derechos humanos. Pero todavía hay mucho por hacer en el ámbito de la promoción, por parte del Movimiento Olímpico, del deporte y los derechos humanos. Teniendo en cuenta el prestigio del COI y los medios de los que dispone, así como su situación como miembro observador de las Naciones Unidas, podría ejercer su influencia, para enfrentar y solventar los abusos sufridos, por ejemplo, por niños deportistas que soportan cargas de entrenamiento demasiado intensivas o ante la discriminación racial en relación con el acceso y el proceso de las competiciones y en actos racistas cometidos por jugadores, deportistas y espectadores.

Palabras clave: Derechos Humanos, Movimiento Olímpico.

Title: The Role of the Olympic Movement in the promotion of Human Rights

\begin{abstract}
:
The United Nations Organisation (UNO) defines human rights as "universal legal guarantees protecting individuals and groups against actions and omissions of governments that interfere with human dignity". However, the behaviour of certain sports organizations often violates these rights. The Olympic Movement must play a role in the promotion and defence of human rights. The Olympic Charter is the only international legal text in the world that expressly recognizes sport as a human right. The 2020 Agenda and regular revisions to the Olympic Charter clearly show the IAO's leadership in promoting and defending human rights. But there is still much to be done in the field of promotion by the Olympic Movement of sport and human rights. Considering the prestige of the IAO and the means available to it, as well as its status as an observer member of the United Nations, it could exercise its influence, to confront and solve abuses suffered, for example, by child sportsmen who bear overly intensive training burdens or in the face of racial discrimination in relation to access to and process of competitions and in racist acts committed by players, athletes and spectators.
\end{abstract}

Keywords: Human Rights, Olympic Movement.

\footnotetext{
${ }^{1}$ El texto del presente artículo es la versión en español del trabajo expuesto por su autor en la $16^{\text {th }}$ International Session for Directors of NOAs celebrada este año de forma telemática, desde la sede de la Academia Olímpica Internacional en Olimpia (Grecia) y que fue presentada el día 11 de septiembre del 2020.
} 


\section{1.- Introducción}

Aunque siempre he seguido los Juegos Olímpicos con entusiasmo, solo a partir de mediados de los años 90 y gracias a la AOI, este entusiasmo se transformó en compromiso con el Olimpismo y con sus valores e ideales. No soy la misma persona, a nivel personal, académico y profesional, desde que tuve la oportunidad de conocer a personas de diferentes países, en la AOI en Olimpia, y pude vivir y aprender junto a ellos ¡Os lo agradezco enormemente!

\section{Los Derechos Humanos: concepto y circunstancias actuales}

Como establece la Carta Olímpica, el Movimiento Olímpico incluye a las organizaciones, los deportistas y otras personas que están de acuerdo con la Carta. La autoridad suprema y máxima del Movimiento Olímpico es el Comité Olímpico Internacional (COI). La Carta Olímpica también identifica los tres elementos principales del Movimiento Olímpico, además del COI; es decir, las Federaciones Internacionales y los Comités Olímpicos Nacionales. En consecuencia, cualquier debate sobre cómo puede el Movimiento Olímpico promover los Derechos Humanos es, en lo esencial, un debate sobre si el movimiento asociativo mundial, es decir, los agentes deportivos de todo el mundo tienen un papel en la promoción y la defensa de los derechos humanos, además del papel que tienen los Estados en este ámbito.

En primer lugar, observemos el contexto.

La Organización de las Naciones Unidas (ONU) define los derechos humanos como «las garantías legales universales que protegen a las personas y los grupos contra acciones $u$ omisiones de los gobiernos que interfieran con la dignidad humana». ${ }^{2}$

Por lo tanto, los derechos humanos son una «serie de cláusulas legales, cuya finalidad es proteger a la persona de los abusos de poder en que incurran los órganos estatales». ${ }^{3}$ En consecuencia, las personas pueden invocar los abusos que cometa un estado, $\mathrm{y}$, concretamente, en el ámbito deportivo, se muestra la relevancia de la relación entre el deporte y los Derechos Humanos.

Sin embargo, los organismos puramente privados, o sea, las organizaciones deportivas, también deben en su comportamiento respetar los Derechos Humanos, aunque sea de forma indirecta. Por ejemplo, y como ya ha dictaminado el Tribunal Arbitral del Deporte ${ }^{4}$ en varias ocasiones, la Convención Europea de Derechos Humanos se aplica a los asuntos disciplinarios de las federaciones deportivas, aunque sea de forma indirecta, a través de la invocación del orden público procesal suizo. También se debe tener en cuenta que, en el reciente juicio del conocido caso Semenya, el propio Tribunal Arbitral del Deporte consideró un informe de amicus curiae presentado por las Naciones Unidas, ${ }^{5}$ es decir, dio la debida atención a la «legislación internacional sobre los Derechos Humanos» para valorar la validez de las normas de una federación deportiva internacional.

Además, los UNGPS, los Principios Rectores de las Naciones Unidas sobre Las Empresas y los Derechos Humanos, que se aplican tanto a los Estados como a las empresas, son afortunadamente y más frecuentemente, tomados en consideración por las organizaciones deportivas, que participan cada vez más e inevitablemente en el mundo de los negocios. La Declaración Tripartita de Principios de la OIT relativa a las Empresas Multinacionales y la

\footnotetext{
${ }^{2}$ Ministério Público Portugal. Departamento Cooperação Judiciária y Relações Intenacionais. O que são os direitos humanos? http://gddc.ministeriopublico.pt/pagina/o-que-sao-os-direitos-humanos

${ }^{3}$ Cabral Barreto, Ireneu (2015), p. 17.

${ }^{4}$ Cf. CAS 2011/A/2426, § 62 et seq ; TAS 2011/A/2433, § 57 et seq ; CAS 2015/A/4304, $\$ 46$ et seq ; CAS 2017/A/5003, § 261 et seq; CAS 2011/A/2384 \& 2386, UCI v Alberto Contador \& RFEC/WADA v Alberto Contador § 22; 2013/A/3139, Fenerbahçe SK v UEFA, §§ 89 e 93; 2013/A/3274, Mads Glaesner v FINA, § 65. ${ }^{5}$ Court Arbitration for Sport. Arbitral Award Delivered by the Court of Arbitration For Sport. 553 et seq.
} 
Política Social, los Principios Rectores de la OCDE (Organización para la Cooperación y el Desarrollo Económicos) ${ }^{6}$ dirigida a las corporaciones multinacionales y la ISO 26000, administrada por SGS, ${ }^{7}$ evalúa el desempeño de las organizaciones y la mejora de la conducta socialmente responsable.

Los organismos privados, en consecuencia, también han «asumido» el respeto por los derechos humanos. De hecho, como ha indicado el Centro para el Deporte y los Derechos humanos, de reciente creación, esto debería entenderse como una tarea: es decir, todas las personas relacionadas con el deporte deben identificar, prevenir, reducir y tener en cuenta el impacto de sus acciones en los derechos humanos, acudiendo a procedimientos de diligencia debida. ${ }^{8}$

Por lo tanto, no puede existir ninguna duda sobre la importancia de la relación entre el deporte y los derechos humanos en la actuación de los estados y de las entidades deportivas. Por lo tanto, debe llegarse a la conclusión de que el Movimiento Olímpico debe tener un papel en la promoción y la defensa de los derechos humanos.

\section{Los derechos humanos en la Carta Olímpica y en los órganos de la estructura del Movimiento Olímpico}

Pero seamos justos. Se ha avanzado mucho, especialmente por parte del COI, entre otras cosas con la inclusión de las siguientes cláusulas en la Carta Olímpica, además del esfuerzo del COI por implantarlas. En primer lugar, la Carta Olímpica, en el acertado texto adoptado en julio de 1996, definió el deporte como un derecho humano, de la siguiente forma:

«La práctica deportiva es un derecho humano. Toda persona debe tener la posibilidad de practicar deporte sin discriminación de ningún tipo y dentro del espíritu olímpico, que exige comprensión mutua, espíritu de amistad, solidaridad y juego limpio». (Cuarto Principio Fundamental del Olimpismo).

La Carta Olímpica es pues el único texto legal internacional del mundo que reconoce expresamente el deporte como derecho humano. Sobre ello, volveremos más adelante.

En segundo lugar, la Agenda 2020 y las revisiones periódicas de la Carta Olímpica, muestran claramente el liderazgo del COI en la promoción y la defensa de los derechos humanos. Por ejemplo, al incluir la defensa del Medio Ambiente, la no discriminación y la justicia en el procedimiento de las solicitudes para acoger los Juegos Olímpicos, además de haber incluido en los nuevos Contratos de Ciudad Anfitriona cláusulas que deben centrarse en el respeto a los derechos humanos. Además, y, por otro lado, hay que mencionar el trabajo del COI en la defensa de los refugiados, o la importancia de Solidaridad Olímpica en el apoyo económico a quienes tienen menos recursos, el combate de la desigualdad y la promoción del desarrollo y la paz. ${ }^{9}$

\section{La problemática actual y la necesidad de evolución}

Pero todavía hay mucho por hacer por parte del Movimiento Olímpico en el ámbito de la promoción del deporte y los derechos humanos y la relación entre ellos.

\footnotetext{
${ }^{6}$ Institute for Human Rights and Business, Championing Human Rights: in the Governance of Sport Bodies, at https://www.ihrb.org/focus-areas/mega-sporting-events/championinghuman-rights-governance-sports-bodies

${ }^{7}$ SGS es la empresa líder en inspección, verificación, ensayos, y certificación

${ }^{8}$ Cf. Sporting Chance Principles on Sport and Human Rights, 2018.

${ }^{9} \mathrm{La}$ «Defensa y el respeto a los derechos humanos» es uno de los requisitos operativos que debe implantar el Comité Organizador de los Juegos Olímpicos, conforme a los «Requisitos operativos de los contratos de las ciudades anfitrionas» (junio de 2018)
} 
De hecho, también existen casos lamentables de discriminación, que afectan principalmente a los sectores más vulnerables de la sociedad; es decir, a grupos marginados con una situación personal limitada, quienes, por diferentes razones, se enfrentan a muchos obstáculos. Por ejemplo, sigue habiendo casos en los que se limita el acceso de las mujeres a la práctica deportiva, en todos los niveles. Todavía se prohíbe a algunas mujeres acceder a estadios deportivos como espectadoras. Existe una diferencia económica entre hombres y mujeres, tanto en cuanto a los salarios como en los premios. Algunos deportes femeninos no están incluidos en la programación deportiva estándar, aunque esté incluido el deporte masculino equivalente. ¿Qué ocurre con el caso Semenya?

Considero que las normas de la Asociación Internacional de Federaciones de Atletismo $(\mathrm{IAAF})^{10}$ vulneran el derecho a practicar deporte, limitando el derecho de determinadas mujeres a participar en algunas competiciones, aunque no en todas: las competiciones de $400 \mathrm{~m}, 800 \mathrm{~m}$ y $1500 \mathrm{~m}$, de forma discriminatoria, arbitraria y desproporcionada. No se justifica que la ética deportiva y la conservación de la integridad de la competición sean, claramente y por sí mismas, justificación para estas limitaciones. Me parece un caso de discriminación, no participar en determinadas competiciones por razón de sexo o género (como se distingue para fines deportivos) por normas que se centran en el derecho de las mujeres (o mujeres con determinadas características fisiológicas), por ejemplo, mujeres con hiperandrogenismo femenino.

También existe la discriminación en base a la discapacidad. Por ejemplo, las organizaciones deportivas que se niegan a permitir a las personas discapacitadas entrenar o participar en deportes recreativos o de competición por incumplimiento en la legislación en relación con el acceso. Por ejemplo, evitando el acceso a instalaciones deportivas a personas con movilidad reducida o limitada, y no garantizar la seguridad y la comodidad de estas personas; diferencias salariales; diferentes niveles de cobertura mediática de los diferentes eventos...

Además, muchos niños son víctimas de abusos sexuales, soportan unas cargas de entrenamiento demasiado intensivas y trabajo forzado, por no hablar del tráfico de niños y niñas en relación con el deporte.

Continúa existiendo discriminación racial en relación con el acceso y en el proceso de las competiciones y actos racistas cometidos por jugadores, deportistas y espectadores.

Es incomprensible que la discriminación en base a la orientación sexual, o contra la comunidad LGBTI en general y contra jugadores y deportistas transexuales, siga proliferando en el siglo XXI.

Igualmente, la normativa deportiva incumple el derecho a la nacionalidad («nacionalidad legal» frente a «nacionalidad deportiva»), el derecho a la privacidad (en casos de dopaje y controles de dopaje fuera del contexto de las competiciones y el sistema whereabouts $\mathrm{o}$ del paradero del deportista). Hay normas de clubs que vulneran el derecho de los jugadores y los deportistas a la vida privada y/o la dignidad humana, con frecuencia en base a la salud pública, la ética deportiva y la integridad.

Por ejemplo, los jugadores y los deportistas siguen estando privados de sus derechos fundamentales (a veces, de forma arbitraria y desproporcionada). La libertad de expresión de los jugadores, deportistas y espectadores se limita para proteger el legítimo interés deportivo y comercial de los organizadores de eventos deportivos, para proteger las «propiedades olímpicas» o combatir fenómenos como la publicidad no autorizada.

\footnotetext{
${ }^{10} \mathrm{La}$ cuestión es la exigencia de que atletas como Semenya, que tienen diferencias de desarrollo sexual, que son mujeres biológicamente, pero con un desarrollo sexual atípico (hiperandrogenismo) tomen medicación para reducir sus niveles naturales de testosterona, para tener derecho a participar en determinadas competiciones deportivas a nivel internacional.
} 
La organización de algunos de los principales eventos deportivos a veces supone trabajo infantil y la muerte de trabajadores como consecuencia de sobrecarga y la ausencia de normas técnicas, sanitarias y de seguridad. A veces implica el desplazamiento de propietarios de tierras dentro de las instalaciones deportivas y la imposición de restricciones a los periodistas en relación con las críticas a las ciudades anfitrionas de eventos deportivos.

Además, la prohibición de prendas con connotaciones religiosas, como el hijab, puede implicar una vulneración de la libertad de pensamiento, conciencia y religión, en ausencia de motivos relacionados con la seguridad de los jugadores y deportistas, o el uso igualitario de equipamientos y materiales deportivos.

Del mismo modo, prácticas como la obligación de que los deportistas o jugadores entrenen por separado equivalen a acoso, que afecta al derecho al trabajo, que también se ve vulnerado cuando se imponen medidas disciplinarias desproporcionadas o cuando se bloquean los traspasos sin justificación.

También se dan casos de intimidación y/o detención de defensores de los derechos humanos cuando se reúnen con intención de manifestarse, lo que puede implicar una vulneración de la libertad de reunión, o normas que exigen la creación de asociaciones, como requisito previo para obtener algún derecho o apoyo, lo que supone problemas en relación con la libertad de asociación.

Además, los jugadores a veces se ven obligados a engañar, a hacer trampa y a manipular los resultados de partidos en los que participan, bajo pena de consecuencias graves, lo que implica un trato degradante y vulnera la prohibición de tortura. Del mismo modo, sigue habiendo ocasionalmente casos de seguidores agredidos por vestir los colores de un equipo rival...

Finalmente, la «justicia deportiva», los mecanismos de resolución de controversias de las federaciones deportivas y el arbitraje deportivo no siempre respetan el derecho de acceso a los tribunales, un principio estándar de las constituciones de los estados democráticos, cuando prohíben totalmente acudir a los tribunales ordinarios, o cuando este acceso a la «justicia deportiva» es demasiado costoso. También existen casos relacionados con la responsabilidad directa de deportistas, que vulneran la presunción de inocencia.

\section{Conclusiones}

Este diagnóstico debe llevarnos a la conclusión de que el Movimiento Olímpico debería, combatir, «desde arriba hasta abajo» (el COI, los deportistas y otros agentes implicados), todas estas lacras que todavía persisten, con normas que debería adoptar en su trabajo y en sus actividades.

Para finalizar, me gustaría hacer algunas sugerencias, que se centran principalmente en el papel del COI, como autoridad principal del Movimiento Olímpico, y, por lo tanto, también como líder de la lucha de este Movimiento por promover y defender los derechos humanos:

1. Teniendo en cuenta el prestigio del COI, pero también los medios de los que dispone y su situación como miembro observador de las Naciones Unidas, podría ejercer su influencia para convencer a los Estados para que incluyan de forma expresa el deporte en sus Constituciones como derecho fundamental, así como también en los textos de derecho internacional público, principalmente en la Declaración Universal de los Derechos Humanos. Esto reforzaría el deber de los Estados de garantizar la promoción y el respeto por los derechos humanos, en y a través del deporte; 
2. Los diferentes deportes dependen, en gran medida, del papel de las Federaciones Internacionales y de los Comités Olímpicos Nacionales, que son elementos fundamentales del Movimiento Olímpico. Podría ser adecuado que el COI actualice su documento de 2013 en relación con el reconocimiento de las Federaciones Internacionales, añadiendo criterios relacionados con la protección y promoción de los derechos humanos ( Federaciones deportivas internacionales que soliciten el reconocimiento del COI: procedimiento de reconocimiento», 2013), y seguramente llevar a cabo una evaluación pública de las actividades de las Federaciones Internacionales que ya hayan sido reconocidas. Ello, además, podría incluir la concesión de clasificaciones o premios relacionados con la calidad, y una mayor financiación, entre otras medidas, para reforzar la lucha fundamental contra la vulneración de los derechos humanos;

3. Teniendo en cuenta que las actividades del Movimiento Olímpico también deben contemplar al público en general, y como la opinión pública es fundamental, sería positivo que el COI explicase y promoviese debates en relación con la necesidad y la proporcionalidad de las restricciones de los derechos humanos en la defensa de la ética deportiva y la singularidad del deporte. Por ejemplo, en la actualidad se está debatiendo en todo el mundo la cuestión de las Normas 40 y 50 de la Carta Olímpica. Además, el activismo social y político de los deportistas es cada vez más visible y perceptible. Es vital que aumenten el diálogo y la comunicación con el público en general, o el Movimiento Olímpico corre el riesgo de que el público en general se forme la idea de que esta institución, que combate las vulneraciones de los derechos humanos, también los vulnera.

Esta es mi modesta opinión.

\section{5.- Bibliografía y fuentes}

- Cabral Barreto, Ireneu (2015). A Convenção Europeia dos Direitos do Homem, Coimbra, Almedina. Portugal.

- Court Arbitration for Sport. Arbitral Award Delivered by the Court of Arbitration For Sport. CAS 2018/0/5794 Mokgadi Caster Semenya v. International Association of Athletics Federations CAS 2018/0/5798 Athletics South Africa v. International Association of Athletics Federations Cf. CAS 2018/O/5794 \& CAS 2018/O/5798, § 553 et seq.

— International Olympic Committee (2018). Host city contract operational requirements Lausanne (Switzerland).

— International Olympic Committee (2016). The Olympic Charter. Lausanne (Switzerland).

- Ministério Público Portugal. Departamento Cooperação Judiciária y Relações Intenacionais. $O$ que são os direitos humanos? http://gddc.ministeriopublico.pt/pagina/o-que-sao-osdireitos-humanos 